\title{
Aplicación de un Ciclo de Mejora de la Docencia en la asignatura Bioquímica Humana
}

\author{
Amalia RuBio Calvo \\ Departamento de Bioquímica Médica y \\ Biología Molecular e Inmunología \\ Facultad de Medicina \\ Universidad de Sevilla \\ amaliarubio@us.es \\ D.0.I.: http://dx.doi.org/10.12795/JDU.2018.i01.39 \\ Pp.: 695-713
}

\section{Resumen}

Se presenta una experiencia de mejora en la asignatura Bioquímica Humana en el Grado en Bioquímica, en concreto en dos temas teóricos de la asignatura. En este trabajo se propone un cambio en el modelo metodológico anteriormente basado en la clase teórica magistral. El modelo propuesto es un aprendizaje por preguntas y problemas que los alumnos deben ir resolviendo en grupo elaborando por si mismos el tema a tratar. De esta forma se cambia de un modelo unidireccional a un modelo más participativo y de trabajo autónomo, donde el docente no tiene ya un papel tan central. Los resultados han sido muy positivos ya que ha mejorado la participación de los alumnos. El análisis de los cuestionarios inicial-final me ha proporcionado una información importante de la evolución de los alumnos tras el trabajo en clase, y también de los conocimientos iniciales de los que partían.

Palabras clave: Bioquímica Humana, Grado en Bioquímica, Docencia Universitaria, Experimentación Docente Universitaria, Envejecimiento 


\section{Breve descripción del contexto}

Los Ciclos de Mejora Docente se han aplicado en la asignatura de tercer curso "Bioquímica Humana" del Grado en Bioquímica. Esta es una asignatura optativa que consta de 6 créditos (35h de clases teóricas, 8h seminarios teórico-prácticos, $11 \mathrm{~h}$ seminarios en los que los alumnos exponen un tema en relación a la asignatura y 6 h de prácticas de laboratorio). Este es un curso que tiene la particularidad de tener un número reducido de alumnos (50 alumnos) que entran en el grado con una nota de corte elevada. Son alumnos muy preparados, con muy buen nivel a los que les interesa la asignatura, aunque son muy poco participativos generalmente.

\section{Diseño previo del Ciclo de Mejora}

\section{Experiencia previa del primer Ciclo de Mejora}

El primer Ciclo de Mejora de la Docencia llevado a cabo en este curso me hizo ver que otra aproximación a la docencia era posible. En esta primera experiencia cambié la metodología que normalmente seguía en clase: explicación del tema mediante clase magistral, y al finalizar la misma o en otra clase realización de preguntas teórico-prácticas y/o planteamiento de casos clínicos relacionados. En esta ocasión impartí la docencia al revés, primero les planteé un caso clínico que trabajaron por parejas y que después debatimos entre todos aportándoles más información por mi parte cuando era necesario. En la elaboración de este primer ciclo fue importante la lectura del capítulo 5 de la obre de Ken Bain (Bain, 2004), y particularmente fue muy beneficiosa la recomendación de elegir una pregunta inicial que captara el interés de los alumnos. En este caso el tema era "Metabolismo del Etanol" y la pregunta inicial que planteé fue: 
"A mí no me hace daño el alcohol, yo estoy acostumbrado a beber, lo metabolizo rápido" ¿Es cierta esta afirmación? ¿En que se basa?

La clase funcionó muy bien con el diseño elegido. Los alumnos participaron más que nunca y realmente aprendieron porque fue respondiendo a todas las preguntas que realmente se hacían ellos y no tanto a lo que yo pensaba que debían saber.

Tras la experiencia del primer Ciclo de Mejora, diseñé un segundo ciclo en el que me planteé objetivos más ambiciosos:

1. Una mejor estructuración de los contenidos en el tema a impartir elaborando un mapa de contenidos

2. Elaboré un cuestionario inicial y final con el fin de conocer los conocimientos previos del alumno y seguir la evolución de los mismos.

3. Diseñe una serie de actividades y preguntas encadenadas que debían trabajar en grupo y que debatiríamos al finalizar la clase. En este diseño mi papel en el aula dejaba de tener protagonismo para otorgárselo a los propios alumnos.

El tema elegido fue “Envejecimiento", el último tema del programa de la asignatura Bioquímica Humana. El tiempo disponible para llevar a cabo la sesión era de 2 horas.

\section{Mapa de contenidos.}

Para este tema elaboré un mapa de contenidos en el que destaqué los contenidos conceptuales, los procedimientos y los contenidos actitudinales (figura 1). 
¿ES POSIBLE REALIZAR INTERVENCIONES ANTIENVEJECIMIENTO?

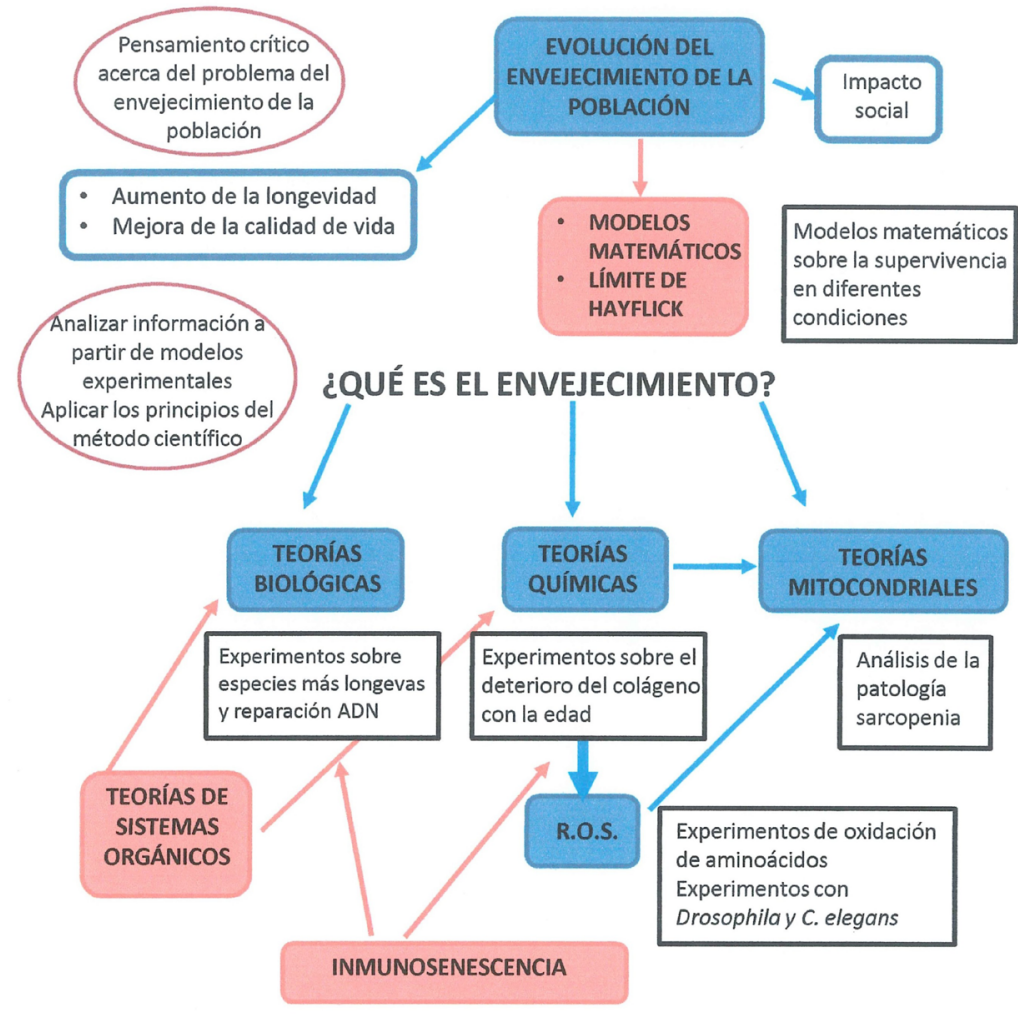

MODELOS ANTIOXIDANTES Y DE RESTRICCION CALORICA DEL AUMENTO DE LA ESPERANZA DE VIDA

Figura 1. Mapa de contenidos. Tema Envejecimiento. Leyenda:

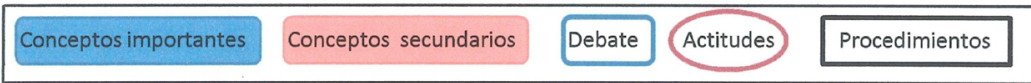

Figura 1. Mapa de contenidos

La realización del mismo me ha hecho reflexionar sobre los diferentes aspectos de los contenidos que generalmente imparto. Siempre he tenido claro que impartía conceptos pero no había reparado que en la enseñanza de dichos conceptos iba implícita la enseñanza de procedimientos y también determinas actitudes. También me ha ayudado a establecer diferencias entre la importancia de contenidos. Así establecí dos niveles de contenidos, 
aquellos que consideraba esenciales y los que eran más secundarios. A la hora de elaborar el cuadernillo de trabajo para realizar en el segundo Ciclo de Mejora tuve en cuenta esta jerarquía en cuanto a la importancia de los contenidos.

\section{Cuestionario inicial y final}

En este ciclo de mejora, elaboré también un cuestionario en el que los alumnos demostraran sus conocimientos previos sobre el tema y que me permitiera analizar su capacidad de razonamiento con base científica acerca de las cuestiones planteadas. Intenté que las preguntas despertaran su interés como en el primer ciclo de mejora. Las preguntas fueron las siguientes:

1. Enumere los tres factores más importantes que considere están implicados en el proceso del envejecimiento.

2. Especule brevemente por qué algunas especies sobreviven más que otras.

3. ¿Cree que es posible retrasar u evitar el envejecimiento de nuestro organismo? ¿Por qué?

\section{Modelo metodológico posible y secuencia de actividades}

El modelo metodológico propuesto es una serie de actividades encadenadas en las que se plantean resultados de experimentos científicos y se pregunta acerca del mismo con el fin de razonar sobre el tema e ir acercándonos a las respuestas de las preguntas del cuestionario inicial. El trabajo se realiza en grupos de cuatro. En el cuadernillo de preguntas se especifica el tiempo estimado que deben dedicarle a cada pregunta y se les sugiere que se nombre una persona que escriba las respuestas, otra que controle el tiempo y otra que haga de portavoz en el debate. Cada grupo debe consensuar una respuesta para cada pregunta. Si fuese necesario aportaré información o 
explicación adicional. Por último se pondrán en común y se debatirán las respuestas de cada grupo consensuando conclusiones. Para la elaboración de este modelo metodológico me he inspirado en el capítulo 6 libro de Don Finkel (2008), "Experiencias que enseñan. Crear esquemas para el aprendizaje". En este capítulo se plantea la elaboración de una ficha de trabajo donde se parte de una primera pregunta suficientemente interesante para que enganche a los alumnos, seguida de una serie de preguntas consecutivas para seguir el problema inicial y que el alumno compruebe su razonamiento y estabilice ideas.

\section{Aplicación del Ciclo de Mejora Docente}

En primer lugar y antes de comenzar la sesión les distribuí el cuestionario inicial con las tres preguntas anteriormente expuestas para que lo contestaran de forma individual. Como era ya la segunda sesión donde experimentábamos otro tipo de docencia y los alumnos ya estaban al tanto de cómo iba a organizarse la clase, el comienzo fue rápido. Se organizaron en grupos de 3-4 alumnos para trabajar con los cuadernillos de trabajo acerca del envejecimiento para lo que dispondrían de 70 minutos. Las preguntas iban enfocadas a que los alumnos razonaran acerca de qué factores, desde el punto de vista genético, bioquímico o celular, estaban implicados en el proceso de envejecimiento (Anexo I). Les fui planteando los resultados de diferentes experimentos de forma que el razonamiento los fuera forzando a tomar partido por una teoría del envejecimiento y a continuación por la contraria. De forma que finalmente los alumnos llegaran a la conclusión que el envejecimiento es un proceso multifactorial en el que probablemente están implicados tanto procesos químicos como biológicos. Por último les planteé los resultados de experimentos con modelos animales para que reflexionaran sobre la posible manipulación del proceso de envejecimiento para retrasar su aparición. Los alumnos trabajaron 
forma autónoma y mi intervención fue más bien escasa en el trabajo en grupo. Al finalizar esta fase de la sesión, pasamos a poner en común las respuestas.

Los alumnos no fueron especialmente participativos y se limitaron a defender sus respuestas o contestar a alguna pregunta que formulé para animar el debate. Han sido tímidos en las respuestas y no se generó mucho debate, quizás este tema les despertaba menor interés. Por otro lado, los alumnos me comunicaron su preocupación acerca de la evaluación, ya que al haberlo abordado el tema de forma totalmente práctica, sin una "teoría" al uso estaban un poco desconcertados acerca de lo que debían saber.

Cuestionario inicial y final (escaleras de aprendizaje)

El cuestionario inicial me mostró que muchos alumnos tienen ya conocimientos previos sobre el tema y que son capaces de establecer un razonamiento científico importante. Son alumnos de tercero, con un bagaje de bioquímica importante, y muchos de ellos fueron capaces de establecer relaciones con su conocimiento anterior. En relación a las respuestas, las subdividí en tres grandes grupos:

1. Razonamiento con argumentos científicos acercándose bastante a lo que espero de la respuesta

2. Razonamiento con argumentos científicos aunque el alumno divague a veces y quedandose en aspectos más simplistas

3. El alumno no utiliza argumentos científicos y razona en base a su sentido común.

Las preguntas iban avanzando en complejidad por lo que también fue disminuyendo el porcentaje de respuestas en el escalón más alto de la escalera de aprendizaje. De cualquier forma, como son buenos alumnos fui bastante exigente a la hora de clasificar los grupos y en las preguntas 2 y 3 solo he agrupado en el escalón 1 a aquellos alumnos que razonaban la respuesta con mayor rigor científico. 
Tabla 1. Evaluación inicial

\begin{tabular}{|l|l|l|l|}
\hline \multicolumn{1}{|c|}{ Preguntas } & \multicolumn{1}{c|}{$\mathbf{1}$} & \multicolumn{1}{c|}{$\mathbf{2}$} & \multicolumn{1}{c|}{$\mathbf{3}$} \\
\hline Grupo 1 (\%) & 39,02 & 14,63 & 7,32 \\
\hline Grupo 2 (\%) & 34,15 & 41,46 & 56,10 \\
\hline Grupo 3 (\%) & 26,83 & 43,90 & 36,59 \\
\hline
\end{tabular}

Una vez terminada la sesión los alumnos contestaron la encuesta de nuevo en casa ya que no dio tiempo al final de la sesión. Por ello, no sé si consultaron libros o en internet o respondieron con lo que habían aprendido en clase. En general las respuestas mejoraron mucho. En muchos casos me han puesto razonamientos que salieron en la clase durante el debate por lo que creo que el aprendizaje fue efectivo.

Tabla 2. Evaluación final

\begin{tabular}{|l|l|l|l|}
\hline \multicolumn{1}{|c|}{ Preguntas } & \multicolumn{1}{c|}{$\mathbf{1}$} & \multicolumn{1}{c|}{$\mathbf{2}$} & \multicolumn{1}{c|}{$\mathbf{3}$} \\
\hline Grupo 1 (\%) & 78,00 & 73,00 & 51,20 \\
\hline Grupo 2 (\%) & 22,00 & 24,30 & 43,90 \\
\hline Grupo 3 (\%) & 0,00 & 2,40 & 4,87 \\
\hline
\end{tabular}

La evolución en general en todas las preguntas ha sido positiva. El porcentaje del escalón más bajo (grupo 3) ha disminuido drásticamente en todas las preguntas, siendo más evidente en las preguntas 2 y 3 en las que se ha pasado de un $43,90 \%$ a un $2,40 \%$ y de un $36,59 \%$ a un $4,87 \%$ respectivamente. La mayoría de los alumnos han superado el nivel superior (grupo 1) siendo superior al $70 \%$ en las dos primeras preguntas.

Es importante destacar también que muchos alumnos aumentaron más de un escalón de una vez sobre todo en la pregunta 2 como se ve en la tabla siguiente. También resaltar que aunque algunos no suban de nivel es porque ya partían de un nivel alto.

Ejemplos de evolución de escalera de conocimientos de los alumnos. 


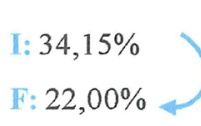

Razonamiento con argumentos científicos aunque el alumno div a gue a veces y quedándose en aspectos más simplistas

I: $39,02 \%$

F: $78,00 \%$

Razonamiento con argumentos científicos acercándose bastante a lo que espero de la respuesta

El alumno no utiliza argumentos científicosy razona en base a su sentido común

Figura 2. Pregunta 1: Enumere los tres factores más importantes que considere están implicados en el proceso del envejecimiento.

Tabla 3. Porcentaje de alumnos que pasó de uno o más niveles en la escalera de conocimiento tras el cuestionario final

\begin{tabular}{|l|l|l|l|}
\hline \multicolumn{1}{|c|}{ Preguntas } & \multicolumn{1}{c|}{$\mathbf{1}$} & \multicolumn{1}{c|}{$\mathbf{2}^{*}$} & \multicolumn{1}{c|}{$\mathbf{3}$} \\
\hline No subió de escalón (\%) & 12.2 & 19.51 & 31.7 \\
\hline Subió un escalón (\%) & 29.00 & 29.68 & 46.34 \\
\hline Subió dos escalones (\%) & 22.00 & 36.58 & 17.07 \\
\hline Ya estaba en el escalón superior & 36.58 & 12.19 & 4.87 \\
\hline
\end{tabular}

\section{Evaluación del diseño puesto en práctica}

\section{Cuestiones a mantener y cambios a introducir}

El modelo metodológico desarrollado durante estos ciclos de mejora ha sido fruto de la reflexión a partir de la participación en los cursos de formación docente, con el apoyo bibliográfico del libro de Porlán y colaboradores 
(2017). El ciclo de mejora ha resultado en general muy satisfactorio ya que me ha acercado a los alumnos haciendo la docencia mucho más participativa, en un grado donde, a pesar de la motivación de los estudiantes, estos no suelen intervenir mucho en clase. Los cambios en los modelos docentes son procesos complejos que dependen de múltiples factores, por lo que aun me queda recorrido en la mejora para llegar al modelo metodológico deseado. En este sentido alguna intervención ha sido más satisfactoria que otra en parte por falta de tiempo en el abordaje de las tareas que quizás fue demasiado ambicioso en relación a los contenidos. Por otro lado, queda también por mejorar la implicación del alumnado en su propio aprendizaje ya que están habituados a una enseñanza unidireccional donde el docente tiene un papel protagonista y trasmisor del conocimiento y he percibido cierto desconcierto e inquietud por como estos nuevos modelos pueden repercutir en su aprendizaje. La utilización del cuestionario inicial y final en la evaluación ha resultado ser una herramienta muy interesante ya que me ha permitido conocer el nivel inicial de conocimientos de los alumnos y sobre todo me ha suministrado una información esencial, cuanto han aprendido realmente en la sesión de trabajo. Este ciclo me ha abierto las puertas a seguir mejorando en estrategias que faciliten la interacción profesor-alumno y el aprendizaje autónomo del conjunto de los alumnos.

\section{Aspectos de la experiencia a incorporar en la práctica docente habitual}

En relación a esta asignatura me planteo incorporar cambios en aquellos temas en los que sea más factible el acercamiento a los contenidos mediante preguntas y análisis de experimentos científicos. Al ser un curso poco numeroso es más factible realizar este tipo de intervenciones. En relación a la docencia en general, tanto si llevo a cabo la clase mediante preguntas o en el formato de clase 
magistral, comenzaré siempre cada tema con una o varias preguntas que despierten el interés del alumno por el tema y fomente la participación. Un reto importante es la disminución de contenidos y la jerarquización de los mismos para lo que me será útil la elaboración de mapas de contenidos. Los cuestionarios iniciales y finales son una herramienta esencial que voy a incluir en la docencia, pero para poder aplicar esta metodología de forma más generalizada debería encontrar una herramienta informática tipo Socrative por ejemplo, que facilitara su realización para que realmente fuera rápido y factible.

\section{Principios didácticos argumentados}

Tras la realización de este curso de iniciación y tras la experiencia en los ciclos de mejora, me planteo los siguientes principios didácticos:

- Elaborar mapas conceptuales y organizar las secuencias de actividades de manera previa al desarrollo de la clase. En estos mapas se resaltarán los aspectos conceptuales, procedimentales y actitudinales a desarrollar para una mejor planificación de la asignatura.

- Previo al comienzo de cada tema se realizará un cuestionario de conocimientos iniciales que volverá a realizarse tras la finalización del mismo para conocer el conocimiento inicial de los alumnos y la mejora del aprendizaje.

- Comenzar cada tema con una pregunta que despierte el interés de los alumnos. El despertar la curiosidad de los alumnos puede generar un cambio sustancial en el desarrollo de la clase.

- Proponer tareas encaminadas al aprendizaje de los contenidos en los que se fomente el trabajo en grupo y la autonomía del alumno. En este sentido guiaré el aprendizaje del alumnado, pero en un plano secundario, con un papel silencioso a la espera de sus preguntas. 
- Dar tiempo de trabajo al alumnado y potenciar su razonamiento crítico.

- Diversificar el tipo de actividades para que el aprendizaje sea ameno y abarque diferentes aspectos desde los conceptuales a los procedimentales y actitudinales.

- Fomentar el debate y la participación a partir de las cuestiones planteadas y de las preguntas que se hagan los mismos alumnos en relación al tema.

- Establecer sistemas de evaluación alternativos que mejoren la evaluación continua del alumno y mejorar los sistemas de evaluación del docente por parte del alumno.

\section{Bibliografia}

Bain, K. (2004). Lo que hacen los mejores profesores universitarios. Valencia: Publicaciones Universidad de Valencia.

Finkel, D. (2008). Dar clases con la boca cerrada. Valencia: Publicaciones Universidad de Valencia.

Porlán, R. (2017). Enseñanza Universitaria, Cómo mejorarla. Madrid: Editorial Morata.

Jornadas de Formación e Innovación Docente del Profesorado I № 1 (2018) Esta obra se distribuye con la licencia Creative Commons 


\section{ANEXO I. Cuadernillo de trabajo. Tema Envejecimiento.}

Integrantes:

Para la realización del trabajo dividíos en grupos de 4 personas. El tiempo estimado para el cuestionario es de 70 minutos. Cada grupo debe consensuar una respuesta para cada pregunta. Seleccionad una persona que escriba las respuestas, otra que controle el tiempo y otra que haga de portavoz en el debate. Respetad los tiempos de discusión para cada pregunta.

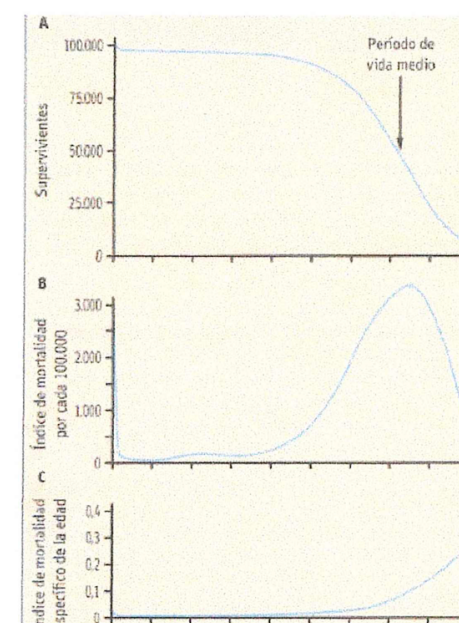

En las 3 gráficas (figura 1) se expresan el índice de supervivencia y mortalidad en función de la edad. El periodo de vida medio se define como la edad en la que sobrevive el $50 \%$ de la población. El índice de mortalidad alcanza el máximo a los 76 años. Las mejoras en los cuidados sanitarios y en el medio ambiente han conducido a una regularización de la curva de supervivencia. Sin embargo ha aumentado nuestro periodo de vida medio pero si un efecto significativo sobre nuestra longevidad máxima

A principio del siglo XIX Gompertz observó que en los humanos el índice de mortalidad específico de cada edad aumentaba exponencialmente después de los 35 años y que las curvas de supervivencia se ajustaban con la siguiente ecuación:
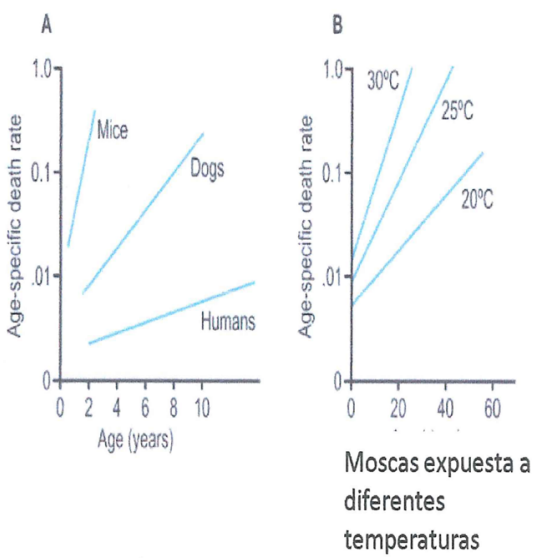
Jornadas de Formación e Innovación Docente del Profesorado I № 1 (2018) Reconocimiento-NoComercial-SinObraDerivada Internacional (CC BY-NC-ND 4.0.) 


\section{Ecuación de Gompertz: $m(t)=$ Aeat}

( $m$ : índice de mortalidad específico de cada edad a la edad t; A: índice de mortalidad al nacimiento; $\alpha$ : el efecto del tiempo sobre el índice de mortalidad, valor contante)

1. Explique según las siguientes representaciones de Gompertz (figura 2) los cambios del indice de mortalidad en diferentes especies y en diferentes condiciones de vida. 10 minutos

2. Se observó además que las moscas encerradas en caja de cerillas viven más que las que se encierran en una bombona de vidrio grande, o que las moscas sin alas viven también más tiempo. Integre esta nueva información en la explicación anterior.

3. Observe la figura 3 donde se observan dos facetas de la inmunosenescencia: un defecto en la señalización y respuesta de las células T, y la involución tímica que genera un menor número de células naive a la circulación sanguínea. En la figura 4 se observa que especies más longevas tienen mecanismos de reparación de ADN más efectivos.
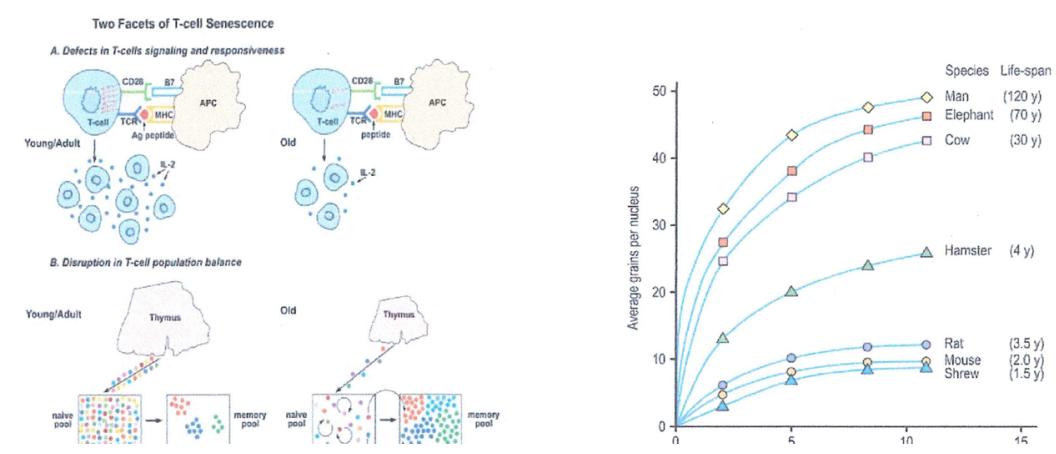

El declive de los sistemas neuroendocrino, reproductor, e inmune, se puede incluir dentro de la actuación de un reloj biológico que afecta las funciones integradas de un organismo. En base a la interpretación de las figuras 
analizadas ¿se podría decir que los procesos de inmunosenescencia son procesos genéticamente programados? ¿Es el envejecimiento un proceso genéticamente programado?

4. La pigmentación es una característica propia del envejecimiento de las proteínas. En la figura 5 se muestran modificaciones de proteínas del cristalino y cartílago costal (proteínas de larga vida) con la edad: se deterioran progresivamente con la edad. Observamos que aumenta el color pardo con la edad debido a la formación de compuestos conjugados que absorben la región rojo-amarilla del espectro

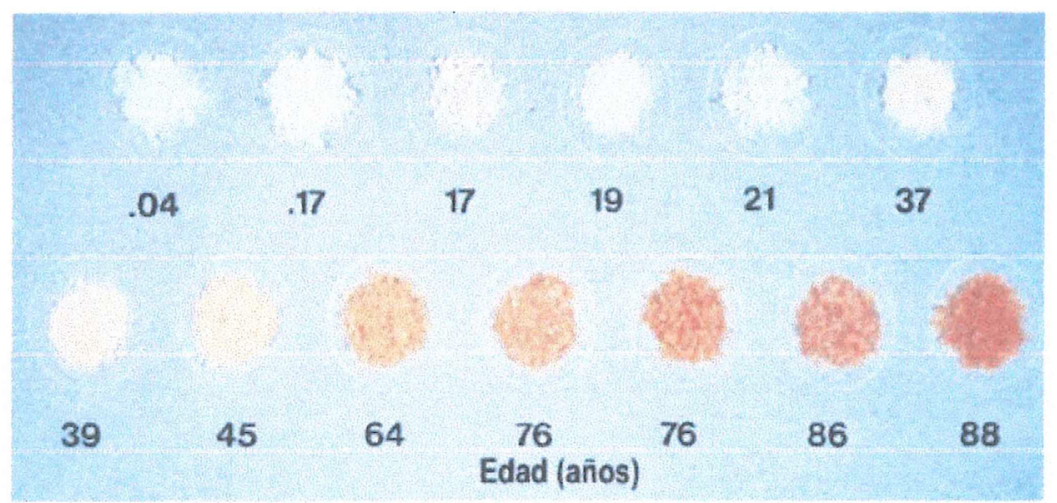

¿Podemos considerar el envejecimiento como una serie de modificaciones químicas crónicas acumulativas que conlleva el deterioro de un número importante de biomoléculas?

5. En las siguientes figuras se muestran otros experimentos en relación con el colágeno. En la figura 6 se observa la acumulación de productos de oxidación de aas con la edad en el colágeno de la piel en humanos. Los organismos de vida larga tienen menores índices de producción de especies reactivas del oxigeno (ROS), mejores defensas antioxidantes y procesos de reparación y recambios más eficientes

Jornadas de Formación e Innovación Docente del Profesorado I № 1 (2018) Esta obra se distribuye con la licencia Creative Commons 

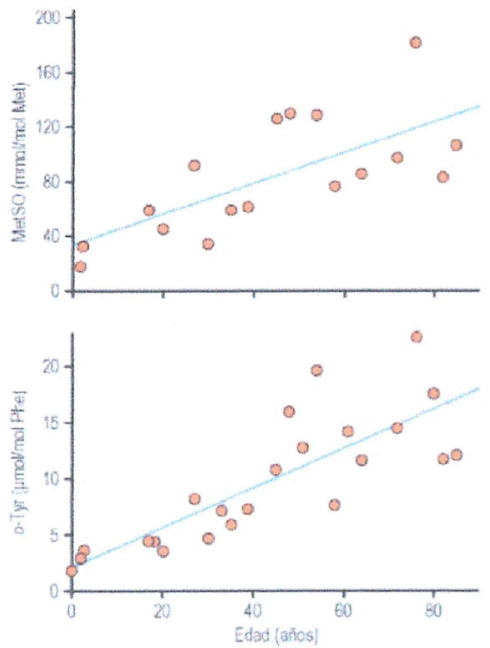

Alteraciones químicas:

Metionina se oxida formando sulfoxido de metionina (MetSO).

Orto Tirosina es producto de de la adición del radical hidroxilo a la fenilalanina.

¿Podemos considerar la acumulación de lesiones oxidativas debidas a las especies reactivas de oxígeno en el ADN, ARN, proteínas, lípidos, y glucoconjugados como la causa de la teoría química del envejecimiento?

6. En relación a la pregunta anterior en la figura 7 se muestran otros daños oxidativos en el colágeno de la piel (en amarillo) y del cartílago (en rojo)
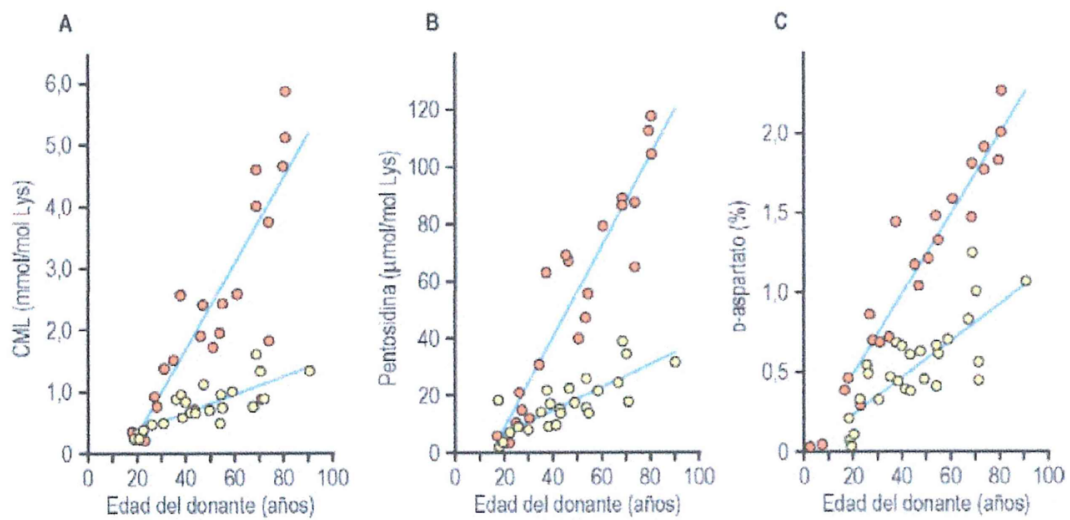

A,B. Acumulación de productos de la glucoxidación y lipoxidación en el colágeno con la edad en humanos. Biomarcadores oxidativos: CML ( $\mathrm{Ne}-$ (carboximetilisina), pentosidina. C. Acumulación la racemización del L-aspartato a D-aspartato. 
Explique si esta figura apoya su razonamiento anterior y explique las diferencias entre las modificaciones encontradas en el colágeno en la piel y en el cartílago

7. En el gusano nematodo Caenorhabditis elegans se ha observado que es importante la participación del componente genético en los mecanismos de la longevidad, como es gen daf-2 relacionado con la respuesta a la insulina. Los mutantes daf-2 pueden vivir el doble de tiempo que los individuos normales siendo estos de menor tamaño. En la figura 8 se observa que experimentos en los que se aplica una restricción calórica a ratones consiguen un aumento en el periodo de vida medio y máximo equivalente a una vida máxima humana de casi 180 años. En la figura 9 se observa que la restricción calórica retrasó la aparición de tumores en ratones. Otros estudios sugieren que la disminución de la masa corporal o la grasa es más importante que la ingestión de calorías. Así los ratones FIRKO (knockout para el receptor de insulina) muestran un descenso de la masa corporal del 15-25\% (disminución del $50 \%$ de grasa). Estos animales comen igual que los controles y observamos un $20 \%$ más de incremento del periodo de vida.

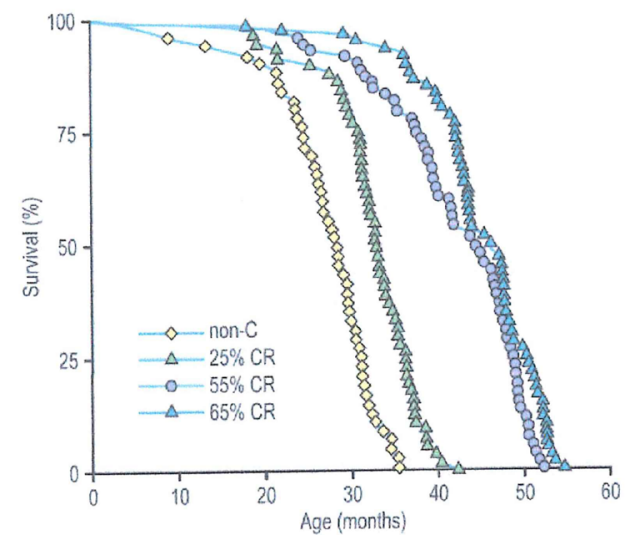

Jornadas de Formación e Innovación Docente del Profesorado | № 1 (2018) Esta obra se distribuye con la licencia Creative Commons 


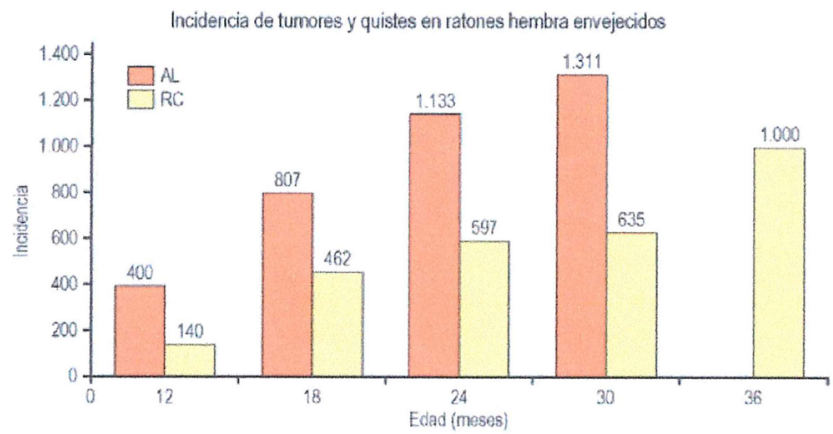

Efecto de la restricción calórica sobre la longevidad de los ratones

¿Es la restricción calórica una intervención antienvejecimiento aconsejable? Justifique su respuesta. Podría tener consecuencias negativas?

Jornadas de Formación e Innovación Docente del Profesorado I № 1 (2018)

(c) (7) $\odot$ Esta obra se distribuye con la licencia Creative Commons 
Jornadas de Formación e Innovación Docente del Profesorado | № 1 (2018) (c) (i) (2) $\odot$ Esta obra se distribuye con la licencia Creative Commons Reconocimiento-NoComercial-SinObraDerivada $\quad 4.0$ Internacional (CC BY-NC-ND 4.0.) 